\title{
Determination of Dimethylamine and Triethylamine in Hydrochloride Salts of Drug Substances by Headspace Gas Chromatography using Dimethyl Sulphoxide- imidazole as Diluent
}

J. GOPALAKRISHNAN* AND S. ASHA DEVI ${ }^{1}$

Piramal Enterprises Limited, Analytical Development Laboratory, 1, Nirlon complex, Off Western expressway, Goregaon (E), Mumbai-400 101, '1School of Biosciences and Technology, VIT University, Vellore-632 014, India

Gopalakrishnan and Asha Devi: Determination of Amines in Drug by Headspace Gas Chromatography

A simple, rapid, accurate and precise analytical method for determination of secondary and tertiary amines in hydrochloride salt form of drug substances in non-aqueous medium, which does not require any derivatization, was developed by headspace gas chromatography. Dimethylamine hydrochloride and triethylamine hydrochloride were analyzed in metformin hydrochloride. The sample was dissolved in a vial containing dimethyl sulphoxide and imidazole. The vials were incubated at $100^{\circ}$, for 20 min. The syringe temperature was maintained at $110^{\circ}$. DB624 column with $30 \mathrm{~m} \times 0.32 \mathrm{~mm}$ i.d., $1.8 \mu \mathrm{m}$ film thickness was used. The injector and detector temperature were $200^{\circ}$ and $250^{\circ}$, respectively. The analytical program used was carrier gas (nitrogen) flow rate: $1 \mathrm{ml} / \mathrm{min}$; injected gas volume: $1.0 \mathrm{ml}$; split ratio: 1:10; oven temperature program: $40^{\circ}$ for $10 \mathrm{~min}$, followed by an increase up to $240^{\circ}$ at $40^{\circ} / \mathrm{min}$. The analytical method was validated with respect to precision, recovery, limit of detection and quantification and linearity.

Key words: Aliphatic amines, dimethylamine, triethylamine, metformin hydrochloride, DMSO, imidazole, headspace gas chromatography

Organic solvents are used in the synthesis of pharmaceutical compounds and they must be controlled to a minimum level in the final product. Their limits are fixed based on their toxicity ${ }^{[1]}$. In general, these residual solvents are analyzed by headspace gas chromatographic technique. Most of the common organic solvents such as toluene, hexane, benzene, chloroform, dichloromethane are chemically nonreactive and does not involve in any chemical reaction during analysis. Selection of suitable diluent for analysis for these types of residual solvents is relatively simple. For these solvents, diluents are selected based on their higher boiling point, their solubility in the sample and the organic solvents to be determined.

Aliphatic amines such as triethylamine and dimethylamine are used in synthesis of many pharmaceutical products. Triethylamine should be controlled to the level of maximum 320 ppmaccording to European Directorate of Quality Medicines Document $(\mathrm{EDQM})^{[2,3]}$. Limit for dimethylamine is not mentioned in any literature. However, as per ICHQ3A (R2), the

*Address for correspondence E-mail: jgk1973@gmail.com limit for unspecified impurities is $0.10 \%$, i.e., 1000 $\mathrm{ppm}^{[4]}$. These amines are chemically reactive in nature and their influence in experimental condition during analysis by headspace gas chromatography was briefly studied by Kott and Chen ${ }^{[5]}$. Various techniques of analyzing organic amines after derivatization using gas chromatography and headspace gas chromatography (GC) techniques were reported by Hiroyuki ${ }^{[6]}$. Influence of $\mathrm{pH}$ of the diluent in aqueous medium during static headspace analysis of aliphatic amines was reported by Maris et al. ${ }^{[7]}$. Analytical method for determination of aniline in water using microwave assisted headspace solid phase micro extraction (SPME) technique was reported by Yan and Jen ${ }^{[8]}$.

This is an open access article distributed under terms of the Creative Commons Attribution-NonCommercial-ShareAlike 3.0 License, which allows other the remix, tweak, and build up to the non-commercially, as long as the author is credited and the new creations are licensed under the identical terms.

Accepted 03 May 2016

Revised 20 Mar 2016

Received 29 Sep 2015

Indian J Pharm Sci 2016;78(3):409-413 
When the drug substance is present in the form of salt, the amines which could be present in the residual amount are also expected to be present in the form of salt. Therefore, it is required to convert the salt form of amine to free base, during headspace gas chromatographic technique. Michael et al. developed analytical method for determination of triethylamine in sarafloxacin hydrochloride on capillary gas chromatography by using glass inlet liners and glass wool which were treated with methanolic potassium hydroxide ${ }^{[9]}$. Raghuram et al. reported quantification of cyclopropylamine, diethylamine and triethylamine in oseltamavir phosphate and candesartan cilexetil by headspace gas chromatography technique. They have treated the active pharmaceutical ingredients in a mixture of aqueous sodium hydroxide and dimethyl sulphoxide and analyzed by headspace gas chromatography ${ }^{[2]}$. Deshpande et al. developed analytical method for determination of diethylamine and triethylamine in oxybutynin hydrochloride and trazadone hydrochloride. They have treated the active pharmaceutical ingredient in a mixture of aqueous sodium hydroxide solution and dimethyl sulphoxide and analyzed by headspace gas chromatography ${ }^{[10]}$.

The aim of the present study is to find out a suitable base which can convert the salt form of amine in to free base in presence of the drug substance in nonaqueous medium. It was found that imidazole was able to convert the salt form of amine in to free base when it was used in non-aqueous medium. The novelty of our study is that imidazole has not been used as a diluent for preparation in any of the residual solvents methods published so far. Metformin hydrochloride was used as the drug substance for the study. Dimethylamine was used as a raw material in the synthesis of metformin hydrochloride ${ }^{[11]}$. Triethylamine is in general used as a base for many organic reactions. A simple, rapid, accurate and precise headspace gas chromatographic technique for analyzing dimethylamine hydrochloride and triethylamine hydrochloride in metformin hydrochloride without any derivatisation was developed.

Triethylamine hydrochloride was obtained from Sigma Aldrich, USA. Triethylamine and dimethyl sulphoxide (DMSO) were obtained from Merck, India. Dimethylamine hydrochloride and imidazole were obtained from S.D. Fine-Chem Limited, India. Metformin hydrochloride was procured from a pharmaceutical company.

Triethylamine hydrochloride $(13 \mu \mathrm{g} / \mathrm{ml}$ of triethylamine) and dimethylamine hydrochloride (25 $\mu \mathrm{g} / \mathrm{ml}$ of dimethylamine) in DMSO were used as standard solutions. Headspace vials for standard were prepared by adding imidazole to the standard solutions. Similarly sample vials were prepared in headspace vials by adding sample, imidazole and DMSO. For determination of triethylamine and dimethylamine, sample size was 200 and $100 \mathrm{mg}$, respectively and imidazole quantity was 0.5 and $1 \mathrm{~g}$, respectively. Blank vials were prepared by adding DMSO in to a headspace vial containing imidazole.

Analytical method validation was carried out with respect to system suitability, linearity, limit of detection (LOD) and limit of quantification (LOQ), precision and accuracy. Specificity of the analytical method was established by injecting individually diluent, standard solutions of dimethylamine hydrochloride and triethylamine hydrochloride in to the chromatograph. For LOD and LOQ, various concentrations of dimethylamine and triethylamine were prepared and injected in to the chromatograph up to their detectable concentration and quantifiable concentration. Precision of the method was determined by injecting six replicate preparations of sample. Accuracy of the method was carried out by spiking the analytes at three different levels, i.e., 50, 100 and $150 \%$ of the specification limit. The samples at each level were prepared in triplicate and injected in to the chromatograph. Linearity of dimethylamine hydrochloride and triethylamine hydrochloride were carried out by injecting standard solutions of these analytes from LOQ to $150 \%$ level of specification limit. Each level was prepared in triplicate and analyzed.

Thermo focus GC was equipped with split/split less injector, column oven, a flame ionization detector, headspace auto sampler (Thermo). This auto sampler was equipped with a glass syringe $(2.5 \mathrm{ml}$ capacity) with temperature controller and vials of standard size and volume of $20 \mathrm{ml}$ capacity. The vial temperature was controlled by using an incubator equipped with automatic mixing system by micro vibration. The vials containing solutions were kept at $100^{\circ}$, for $20 \mathrm{~min}$. Vials were pressurized due to incubator temperature. The syringe temperature was maintained at $110^{\circ}$. DB624 column with $30 \mathrm{~m} \times 0.32 \mathrm{~mm}$ i.d., $1.8 \mu \mathrm{m}$ film thickness was used. The injector and detector temperature were $200^{\circ}$ and $250^{\circ}$, respectively. The analytical program used was carrier gas (nitrogen) flow rate: $1 \mathrm{ml} / \mathrm{min}$; injected gas volume: $1.0 \mathrm{ml}$; split ratio: 1:10; oven temperature program: $40^{\circ}$ for $10 \mathrm{~min}$, followed by an increase up to $240^{\circ}$ at $40^{\circ} / \mathrm{min}$. 
In general, DMSO, N-methyl pyrrolidone, 1,3 dimethyl 2-imidazolidone and dimethyl formamide are the nonaqueous solvents which are being used as a solvent medium for preparation of samples for residual solvents analysis. These solvents are preferred for residual solvent analysis because of their high solubility and high boiling point. Experiments were carried out using all these solvents. It was not possible to convert the salt form of the amine in to free base, using these solvents. Imidazole dissolved in DMSO was preferred because of its basic as well as buffer nature and trials were made. It was found that imidazole in DMSO was able to convert the salt form of amine in to free base quantitatively. Thus, DMSO-imidazole mixture was selected as suitable diluent for this method.

To optimize the quantity of imidazole required for the sample preparation, solutions of triethylamine and triethylamine hydrochloride were prepared separately in DMSO. They were transferred in to headspace vials containing various amount of imidazole. At each level, solutions were prepared in duplicate and analyzed as per the experimental condition. A graph of area response of triethylamine (base and hydrochloride salt) against amount of imidazole used was plotted and shown in fig. 1. The response of triethylamine which was present in the form of triethylamine hydrochloride was very less when less amount of imidazole was used. The response was increasing with respect to increase in amount of imidazole and reached a maximum value. When adequate amount of imidazole was used, the response of triethylamine (both salt and free base) was same and it was not altered due to further addition of imidazole. A similar study was carried out using dimethylamine hydrochloride to optimize the quantity of imidazole and the results are shown in fig. 2. In order to generate the data on $\mathrm{pH}$ of the solutions, the solutions at each stage were mixed with equal amount of water and measured. The results are tabulated in Table 1. When the $\mathrm{pH}$ of the solutions containing free base and salt form of the amine was same, the area response was also same.

Specificity of the method was confirmed as there was no any interference due to diluent at the retention times of the triethylamine and dimethylamine. LOD was determined by calculating the signal to noise ratio with respect to the corresponding noise in the blank injection. Precision at LOQ level was carried out by injecting six different preparations of analytes at their LOQ level and the percentage relative standard deviation (\% RSD) for area was calculated. The results are tabulated in Table 2 .

For linearity study five different concentrations of triethylamine and dimethylamine in the range of 0.37 $19.5 \mu \mathrm{g} / \mathrm{ml}$ and $2.8-37.5 \mu \mathrm{g} / \mathrm{ml}$, respectively were prepared. The vials were prepared in triplicate for each concentration and analyzed. A linear graph was plotted with respect to area response against the concentration of the analytes and their corresponding correlation coefficient, slope and intercept values were calculated. The results are attached in Table 3.

The sample was containing only $0.07 \%$ of

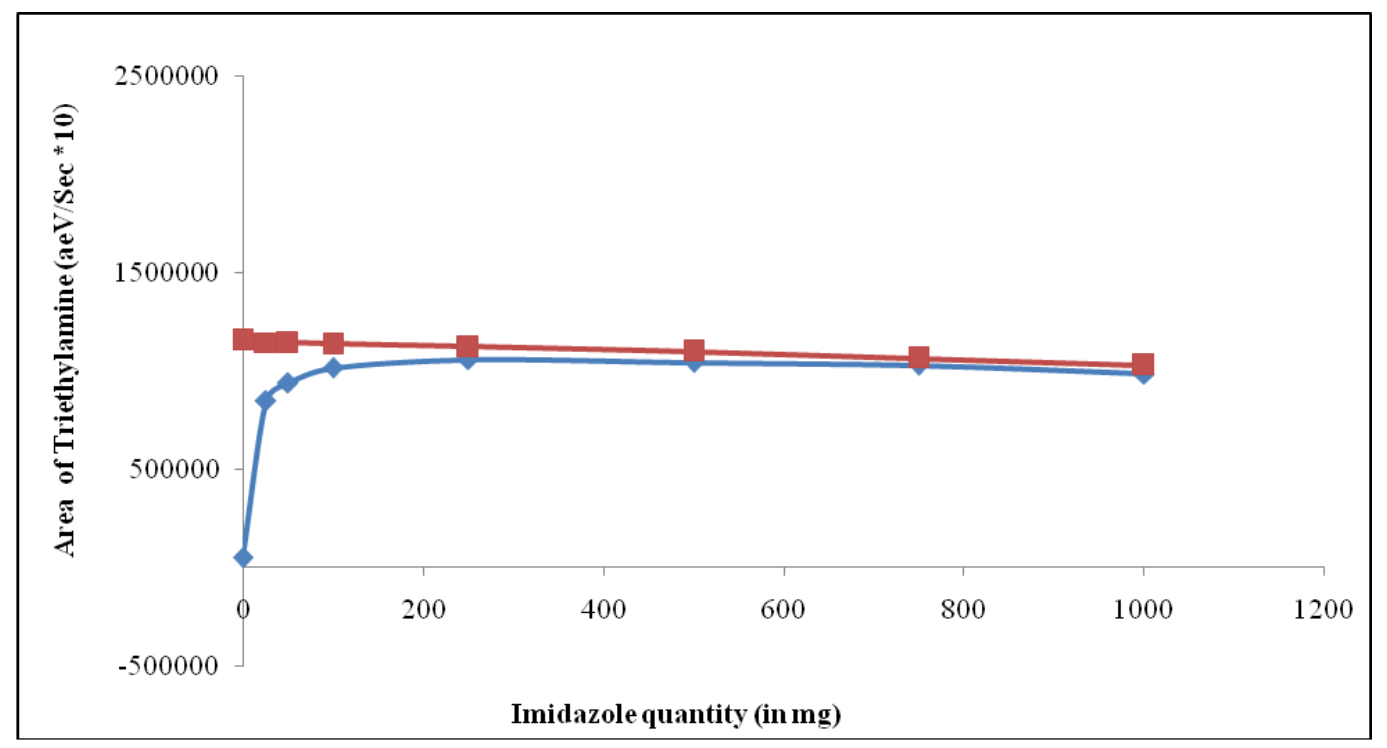

Fig. 1: Triethylamine and Triethylamine hydrochloride response with respect to imidazole quantity. - - TEAHCI; - - - TEA. There was no response observed when triethylamine hydrochloride was analysed at lower $\mathrm{pH}$. At higher pH values, the response of triethylamine and triethylamine hydrochloride by headspace GC was found to be high and it remained same. 


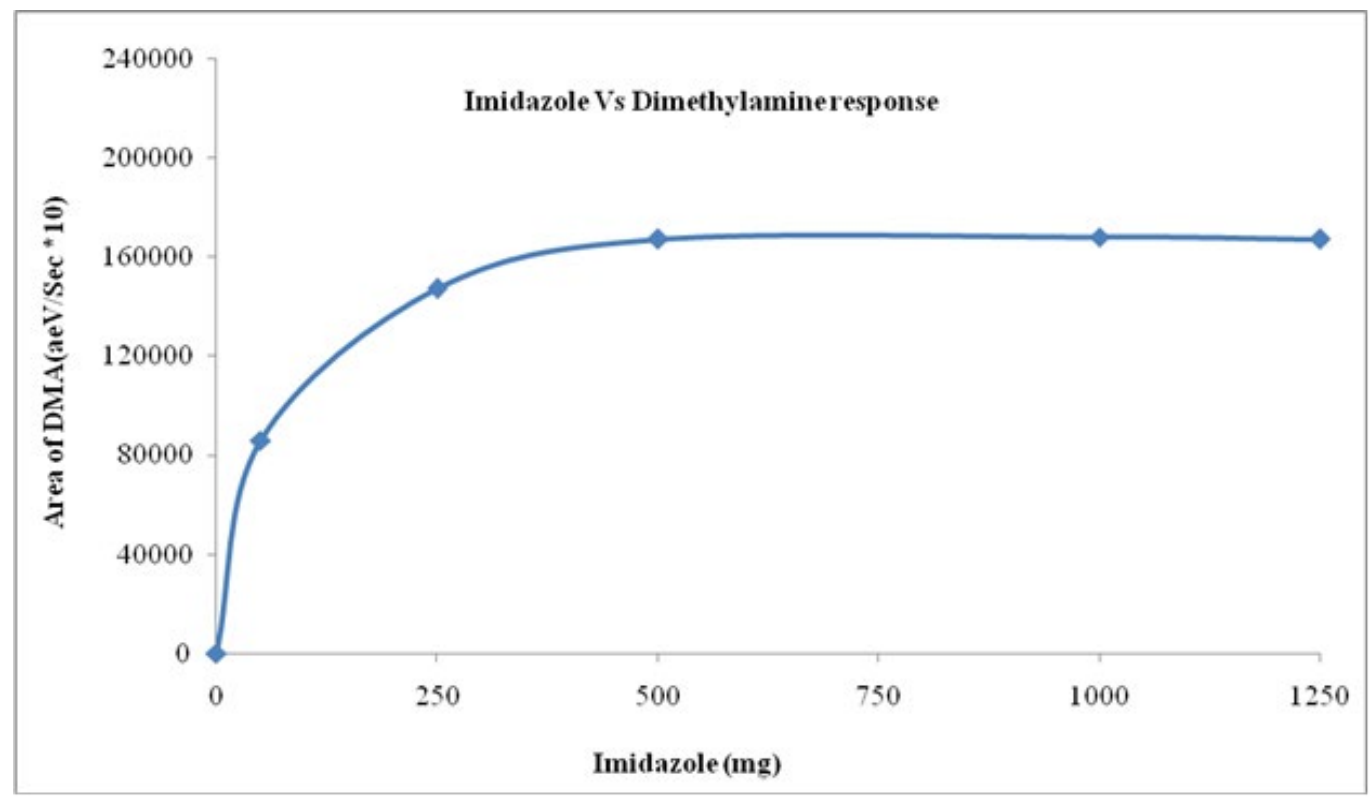

Fig. 2: Dimethylamine hydrochloride response in headspace GC in DMSO-imidazole with respect to imidazole quantity.

The quantity of imidazole required to completely convert the salt form of dimethylamine to free base by headspace GC was determined using this experiment.

TABLE 1: pH OF TRIETHYLAMINE AND TRIETHYLAMINE HYDROCHLORIDE IN DMSO WITH VARIOUS QUANTITIES OF IMIDAZOLE

\begin{tabular}{lccc}
\hline Diluent composition & TEA HCl & TEA & DMA HCl \\
\cline { 2 - 4 } & \multicolumn{3}{c}{$\mathrm{pH}$ (50:50) in water } \\
\hline DMSO (Blank) & & 7.2 & \\
DMSO+1000 mg imidazole (Blank) & 7.5 & 10.1 & 7.2 \\
0 mg imidazole+DMSO & 9.1 & 10.3 & 9.0 \\
25 mg imidazole+DMSO & 9.3 & 10.3 & 9.2 \\
50 mg imidazole+DMSO & 9.6 & 10.2 & 9.5 \\
100 mg imidazole+DMSO & 9.8 & 10.1 & 9.8 \\
250 mg imidazole+DMSO & 9.9 & 10.1 & 9.9 \\
500 mg imidazole+DMSO & 10.0 & 10.0 & 10.1 \\
750 mg imidazole+DMSO & 10.1 & 10.1 & 10.1 \\
\hline g imidazole+DMSO & & & \\
\hline
\end{tabular}

TEA HCl: triethylamine hydrochloride, TEA: triethylamine; DMA HCl: dimethylamine hydrochloride. $\mathrm{pH}$ was checked after adding equal amount of water in to each vial. $\mathrm{pH}$ of the solution was more than 10 when triethylamine was used and it remained same with respect to different quantities of imidazole added to the solution.

\section{TABLE 2: LOD AND LOQ FOR DIMETHYLAMINE AND TRIETHYLAMINE}

\begin{tabular}{lrccc}
\hline Analyte & LOD $(\mu \mathrm{g} / \mathrm{ml})$ & S/N Ratio for LOD & LOQ $(\mu \mathrm{g} / \mathrm{ml})$ & Precision at LOQ $(\%$ RSD $)$ \\
\hline Dimethylamine & 0.93 & 5 & 2.8 & 2.4 \\
Triethylamine & 0.12 & 3 & 0.37 & 4.7 \\
\hline
\end{tabular}

Precision at limit of detection (LOD) and limit of quantification (LOQ) was performed in six replicates.

dimethylamine and triethylamine was absent. Accuracy of the experiment was carried out by spiking 50, 100 and $150 \mu \mathrm{g}$ of dimethylamine in $100 \mathrm{mg}$ of metformin hydrochloride. For triethylamine, accuracy was carried out by spiking 32,64 and $96 \mu \mathrm{g}$ of triethylamine in $200 \mathrm{mg}$ of metformin hydrochloride. $92.5-99.4 \%$ of recovery was obtained for triethylamine and 93.1$104.3 \%$ of recovery was obtained for dimethylamine. Overall recovery at all levels was found to be 97.6 and $96.6 \%$ for dimethylamine and triethylamine, respectively. Overall \% RSD of recovery was found to be $2.4 \%$ and $4.0 \%$ for triethylamine and dimethylamine, respectively.

The proposed method for determination of dimethylamine hydrochloride and triethylamine hydrochloride in metformin hydrochloride is a direct method and it does not involve in any derivatisation. The possible interaction between the analyte, drug 
TABLE 3: LINEARITY STUDY RESULTS FOR TRIETHYLAMINE AND DIMETHYLAMINE

\begin{tabular}{lcc}
\hline Linearity & Triethylamine & Dimethylamine \\
\hline Correlation factor $(r)$ & 0.9975 & 0.9996 \\
Correlation factor $\left(\mathrm{r}^{2}\right)$ & 0.9951 & 0.9991 \\
Slope & 41488 & 6460.7 \\
Y-intercept value & 13452 & -5771.6 \\
$\%$ Y intercept with & 2.7 & -5.3 \\
respect to linearity & & \\
level-4 level & & \\
\hline
\end{tabular}

Linear response for dimethylamine and triethylamine was observed. The \% intercept was found to be below $5 \%$ for both solvents.

substances and the solvent medium and their impact are discussed in detail. The analytical method was validated for precision, linearity, limit of detection, of quantification and accuracy and the results were in acceptable limits. Imidazole was able to convert the salt form of amine in to free base quantitatively and hence it can be used as a diluent in residual solvents for non-aqueous medium. This methodology mentioned in this paper shall be extended for determination of organic volatile amines in the salt for drug substances.

\section{Acknowledgements:}

The authors extend their appreciation to the Head, Pharmaceutical Research and Development, Piramal Enterprises Limited, Mr. Arno Enose for organizing the sample for study and supporting the project.

\section{Financial support and sponsorship:}

Nil.

\section{Conflicts of interest:}

There are no conflicts of interest.

\section{REFERENCES}

1. $\mathrm{ICH}, \mathrm{Q} 3 \mathrm{C}(\mathrm{R} 5)$, Harmonised Tripartite Guideline, Impurities: Guideline for Residual solvents guidelines, in: Proceedings of the International Conference on Harmonization, Geneva, February, 2011.

2. Raghuram P, Soma Raju IV, Sriramulu J. GC quantification of cyclopropylamine, diethylamine and triethylamine in active pharmaceutical ingredients. Chromatographia 2010;71:963-6.

3. EDQM Europe. Content of the dossier for chemical purity and microbial quality.2007; PA/PH/CEP(04) $14 \mathrm{R}$.

4. ICH, Q3A(R2), Harmonised Tripartite Guideline, Impurities in New Drug Substances. In: Proceedings of the International Conference on Harmonization, Geneva, February, 2011.

5. Kott L, Chen HM. Experimental considerations in headspace gas chromatography. Pharm Technol 2010;34:76-9

6. Hiroyuki K. Derivatization reactions for the determination of amines by gas chromatography and their applications in environmental analysis. J Chromatogr A 1996;731:19-34.

7. Maris C, Laplanche A, Morvan J, Bloquel M. Static headspace analysis of aliphatic amines in aqueous samples. J Chromatogr A 1999;846:331-9.

8. Yan CT, Jen JF. Determination of aniline in water by microwave assisted headspace solid phase microextraction and gas chromatography. Chromatographia 2004;59:517-20.

9. Moore WM, Edwards RJ, Bavda LD. An improved capillary gas chromatography method for triethylamine Application to sarafloxacin hydrochloride and GnRH residual solvents testing. Analytical Letters 1999;32:2603-12.

10. Deshpande AR, Ramachandran G, Ramesh SY. Determination of diethylamine and triethylamine quantitatively using GCheadspace chromatography. Eurasian J Anal Chem 2012;7:43-8.

11. Werner E, Bell J. The preparation of methylguanidine and of $\beta \beta$-dimethylguanidine by the interaction of dicyanodiamide and methylammonium and dimethylammonium chlorides respectively. J Chem Soc Transactions 1922;121:1790-5. 